\title{
Acceptance and insight: incapacity to give informed consent
}

van Staden, Werdie (CW)

Correspondence to Professor Werdie (C.W.) van Staden, MBChB, MMed(Psych), MD, FCPsych, FTCL, UPLM, Department of Psychiatry, University of Pretoria, PO Box 667, Pretoria 0001, South Africa Tel: +27 12319 9748; fax: +27 12319 9748; e-mail: werdie.vanstaden@up.ac.za

\section{Outline}

- Abstract

- Introduction

- Causal connection between mental disorder and particular inabilities

- Insight, acceptance and appreciation in assessments of capacity to give informed consent

- Connections between insight and incapacity

- From insight to acceptance in capacity assessments

- Conclusion

- References and recommended reading

\begin{abstract}
Purpose of review: To highlight areas for potential refinement in assessments of capacity to give informed consent.
\end{abstract}

\section{Recent findings:}

The clinical assessment of the patient's capacity to give informed consent may be informed and guided by sophisticated criteria or assessment instruments. The approach of most assessment instruments and the literature on (in)capacity departs from the abilities that underpin giving informed consent. This approach may be refined, however, by assessing clinically for a causal connection between the mental disorder in the mind of a particular person and the particular inability. It may furthermore be refined by assessing that aspect of insight that is best connected to incapacity, for insight has been found to be the best clinical discriminator of capacity status in patients with psychotic and manic disorders.

\section{Summary:}

To find that a person is incapable by virtue of a mental disorder, a causal connection between the mental disorder and the particular inability should be assessed clinically for the very patient. 
Furthermore, the term 'acceptance' is more apt than 'appreciation' and 'belief' in capturing that aspect of insight by which a person with psychotic and manic disorders may be rendered incapable of giving informed consent.

\section{Introduction}

In recent years, there has been a significant shift from a categorical approach to a functional approach in considerations of incapacity to give informed consent [1]. By a categorical approach, the patient is deemed incapable of giving informed consent by virtue of belonging to a particular category such as having a diagnosis of psychosis or dementia [2]. Some authors still see a place in research for a categorical approach, but more in a screening sense, whereby vulnerable people at risk of lacking capacity can be identified [3]. A functional approach requires that a patient's capacity to give informed consent be assessed at the time of giving consent, for a particular decision, and that a patient be examined for particular inabilities or necessary conditions that must pertain for a patient to be considered (in)capable of giving informed consent. [1-3].

The need for a more formal assessment of capacity to give informed consent arises traditionally in the clinical setting for borderline cases and when a patient refuses recommended diagnostic procedures or treatments whether in surgical, medical or psychiatric settings [4]. In the research context, the need for more formal assessments becomes eminent in protection of potential research participants who, by virtue of being incapable of giving informed consent, are at risk of exploitation or harm $[1,5,6,7]$. Some ethics review committees, for example, now require explicit assessment of research participants' capacity for those research protocols that involve more than minimal risk in populations who are considered to be at risk of impaired decisional capacity $[4,8]$.

The assessment of the patient's capacity to give informed consent may nowadays be informed and guided by sophisticated criteria or assessment instruments. No fewer than 29 assessment instruments are available for this purpose [9], of which the MacArthur Competence Assessment Tools for Clinical Research and for Treatment (respectively, MacCAT-CR and MacCAT-T) have the most empirical support $[4,10,11]$. These assess for the four general abilities of understanding, appreciation, reasoning and expressing a choice. These are similar to the abilities identified by the Mental Capacity Act (2005) of England and Wales, namely understanding, retaining, using, weighing and communicating [12].

This review aims to suggest two potential refinements in the clinical assessment of a patient's capacity to give informed consent. These are as follows: to find that a person is incapable by virtue of a mental disorder, a causal connection between the mental disorder in the mind of that person and the particular inability should be assessed clinically; and that the term 'acceptance' be more apt 
than 'appreciation' and 'belief'.

\section{Causal connection between mental disorder and particular inabilities}

The approach of most assessment instruments and the literature on (in)capacity departs from the abilities that underpin decision-making and more specifically valid informed consent $[4,10]$. These abilities should accordingly be of sufficient quality. For example, the understanding of the proposed intervention should be of sufficient extent, and, for this reason, assessment instruments test for the extent of understanding [3]. Similarly, it is assessed how well a person is communicating, reasoning and appreciating.

Although this approach has had much face validity and may have served practical situations well enough in most cases, it holds the risk of overinclusion, that is, rendering people incapable of giving informed consent by setting requirements for informed consent that are not met for good and legitimate reasons, irrespective of having a mental disorder or not. For example, a person may deliberately choose not to understand, or reason about or appreciate the ins and outs of the proposed intervention, for they may find it too upsetting, too grisly, too threatening or even too boring as indeed some research participants suggested [6]. Another example would be that a person might legitimately prefer to trust blindly. For this reason, weaker criteria have been proposed such as having mere capacity to understand rather than actual understanding, and one may argue similarly that it would be sufficient to have mere capacity for the other abilities underpinning decision-making. The weaker criteria, however, may complicate and undermine the validity and the reliability of the assessment of capacity in a given case, as an assessment of capacity to, for example, understand, in general, is likely to be further removed from the specific decision in question.

The former approach departing from the abilities underpinning capacity, in general, and the weaker version thereof may be refined, however, by making the requirement(s) more specific, that is, to require a causal connection between a mental disorder in the mind of a person and the particular inability, as suggested elsewhere [13]. For example, the mental disorder preventing the person to understand, to communicate, to choose, to appreciate, to reason, to weigh and so on.

Requiring this causal connection has three components: the mental disorder in the mind of a person, the particular inability, and the connection. Considering the mental disorder, narrowing it down to those inabilities attributed to mental disorder as qualifying for incapacity, ensures that good and legitimate reasons for not exercising an ability (e.g. understanding) in a particular situation do not render someone 'incapable' (as may be the inappropriate result of attempting to use an assessment instrument of capacity without this specification). Moreover, specifying the pertaining mental disorder makes clear the particular cause of incapacity. The counter argument, however, may be concerned 
that this is too restricted and may ask about causes of incapacity that are not mental disorders. But then, such causes may be specified too as indeed is allowed for by a provision of the Scottish Incapacity Act in stating '... or of inability to communicate because of physical disability' [14]. Aphasia of certain kinds comes to mind.

Connecting causally mental disorder in the mind of a person to particular inabilities also brings more specification in the clinical assessment of the very abilities being assessed. One may argue, for example, that 'actual understanding' is not required for informed consent, but, in case a mental disorder prevents 'actual understanding', it would be difficult, if not impossible, to make a case that the person is capable of giving informed consent. Similarly, one might argue that a person may choose not to communicate or choose to and/or be content with reasoning in contradictory ways, or even choose not to reason at all in making a decision, but if a mental disorder prevents a person from communicating or reasoning in making a decision, it is much clearer that capacity to give informed consent is lacking.

For 'acceptance', the difference brought about by the causal connection is transparent. Ordinarily, when someone does not accept (e.g. some of the information or need for the intervention), they may still agree to the intervention for other reasons and yet be considered capable of giving informed consent [15]. However, when a mental disorder prevents a person from accepting the need for the intervention, it becomes clear that the mental disorder gets in the way of the patient giving informed consent, that is, the person is not capable of giving informed consent even if he says that an intervention may proceed [13]. For example, a patient with a Cotard's delusion believing that she is already dead and electroconvulsive treatment is, for that reason, futile does not accept the need for the treatment by virtue of her mental illness and would not be capable of giving informed consent even when she agrees to the electroconvulsive treatment.

The causal connection between the mental disorder in the mind of a person and the inability may be difficult to demonstrate in some instances, yet be sound in many cases. Examples are: the causal connection between dementia and a lack of understanding fits well, at least conceptually, with particularly cognitive impairment; the indecisiveness of the very ambivalent and depressed person connects causally with being prevented from making a decisive choice. The marked formal thought disorder connects causally with being prevented from reasoning in a sound way. These causal connections are further supported by much empirical research evidence . [8,12,16,17,18,21,22,23].

If the claim is that the incapacity is due to a mental disorder, it has to be accurate that an assessment instrument of incapacity should assess for a causal connection between the mental disorder and the inability. If so specified, the claim is here that the content validity of the assessment 
instrument will be improved, at least for when a mental disorder is present, because the presence of a mental disorder in the mind of a person may or may not have bearing on a particular decision. This means an instrument that assesses for the presence of the causal connection would be able to make a stronger finding of incapacity when a causal connection is indeed demonstrated, or a stronger finding of capacity when there is an absent connection between a chosen lack of, for example, understanding or choice and the mental disorder in the mind of a person.

The first suggested refinement in the clinical assessment of a patient's capacity to give informed consent is thus that a causal connection between the mental disorder in the mind of a person and the particular inability be assessed. Following the suggestion of a causal connection, the second suggested refinement is about one of the abilities that is commonly prevented by psychotic mental disorders and is related to the known clinical concept 'insight', that is, being prevented by the mental disorder from accepting the need for a health intervention.

Insight, acceptance and appreciation in assessments of capacity to give informed consent The clinical concept of insight is of particular importance for capacity assessments in cases of especially psychotic illnesses [18,22], but also for mania [16], yet its connection to incapacity needs to be clear for safeguarding the liberty interests of patients [12].

\section{Connections between insight and incapacity}

The connection between a lack of insight and incapacity has been reported in several recent publications [2,16,19,20,22,24,25,26]. Most convincingly, the study by Owen et al. [12] reports that, among a range of psychopathological variables, insight is the best discriminator of capacity status in patients with psychotic and manic disorders, but less so in nonpsychotic disorders. This was the finding in a cross-sectional study involving 350 patients in which they were assessed for the capacity to consent to hospitalization and treatment, respectively. In another study [16] among patients with mania, a lack of decision-making capacity as assessed in a gambling task was best predicted by a lack of insight among various illness-defining features.

The incapacity linked with a lack of insight goes further than giving informed consent. It involves poor treatment adherence $[27,28,29,30]$, forming a therapeutic alliance [31], and responding to cognitive behavioural therapy $[25,32]$ and fitness to plead . [19].

Following on from the traditional emphasis on the lack of understanding and cognitive impairment in incapacity assessments [4,33], one may ask whether a lack of insight is associated with impairments of cognitive functioning. However, no associations between insight and cognitive functioning have been found in a recent study [34]. Cognitive performance did not discriminate 
capacity status in psychotic disorders, whereas a lack of insight did . [12].

\section{From insight to acceptance in capacity assessments}

Although insight is the clinical concept closest to judgments of incapacity for psychotic illnesses and mania $[12,16]$, insight appears to be too broad a concept that needs specification to make the connection with incapacity clearer $[10,12]$. The insight concept in its psychiatric usage is considered to have the following dimensions: awareness of having a mental illness, awareness of specific signs and symptoms of the disorder, the attribution of symptoms to the mental illness, awareness of the need for treatment and understanding the social consequences of the illness [34]. Similarly, the Expanded Schedule for the Assessment of Insight [35] comprises three main dimensions: awareness of illness, relabelling of symptoms as abnormal and treatment compliance.

Some of these dimensions of insight overlap with the understanding and the reasoning abilities in the MacCAT-T, but it is, in particular, failure of appreciation that is coupled with a lack of insight $[10,18,36]$. The English and Welsh Law Commission favoured the term 'using' instead of 'appreciation' [12]. Also related is whether the person believes (the information) [10,12].

Instead of the terms 'using', 'appreciation' and 'belief', the point I am making in this review is that the term 'acceptance' captures this particular inability better. More specifically, that is, in causal terms, a person being prevented by mental disorder from accepting the need for an intervention [13]. Other than 'acceptance' being a term of clinical importance [37], I suggest the term 'acceptance' is better for the following reasons.

1. The term 'acceptance' is closer to the particular dimension of insight, mentioned above as 'awareness of the need for treatment' [38].

2. The words 'does not accept' feature in the supportive description of the 'appreciation' ability by the designers of the MacCAT [10] (p. 43). This may suggest from the way they use the relevant words that it is more about acceptance than appreciation.

3. In research looking at insight and adherence to medication, it is acceptance that plays a determining role [27] that congruently figures strongly in the Compliance Rating Scale [30].

4. The term 'appreciation' is less desirable than 'acceptance' in that 'appreciation' overlaps considerably with the term 'understanding'. For example, the words 'appreciation' and 'understanding' may be used interchangeably without a change in meaning in many cases. In other cases, one may comprehend 'appreciation' as being 'understanding' in a particular sense.

5. The term 'acceptance' is more specific to the inability in question than 'belief' [13]. For example, the delusional patient may state 'I believe the information you have given me about the proposed treatment, I believe the treatment may be beneficial for some and even for me, but I shall not take it 
because it does not befit me, being royalty from outer space, to take the medicine from common humans'. This is of course notwithstanding that believing may in some cases be specific enough, for, when a mental disorder prevents a person from believing, that would render him or her incapable of consenting. Assessing capacity in terms of acceptance, being more specific than belief, would, however, make an assessment in terms of belief obsolete.

6. The term 'acceptance' is also more specific to the inability in question than the rather general term 'using' of the Law Commission, and which seems to overlap more with the inability of reasoning.

\section{Conclusion}

This review suggested two refinements in the clinical assessment of a patient's capacity to give informed consent. These are that a causal connection between the mental disorder in the mind of a person and the particular inability should be assessed clinically and that the term 'acceptance' be more apt than 'appreciation' and 'belief' in assessments of capacity. When such assessments are done by means of formal instruments such as the MacCAT-T, suggesting these refinements does not question the validity or the reliability of them, but making these refinements to the instruments aims to improve, in particular, the content validity of such instruments. To improve the content validity in this way will be congruent with the calls for further refinement of capacity assessment procedures [36].

\section{References}

1 van Staden CW. Can involuntarily admitted psychiatric patients give informed consent to participate in clinical research? S Afr J Psychiatry 2007; 13:10-12.

2 Appelbaum PS. Assessment of patients' competence to consent to treatment. N Engl J Med 2007; 357:1634-1640.

3 Scott YH, Appelbaum PS, Swan J, et al. Determining when impairment constitutes incapacity for informed consent in schizophrenia research. Br J Psychiatry 2007; 191:38-43.

4 Dunn LB, Nowrangi MA, Palmer BW, et al. Assessing decisional capacity for clinical research or treatment: a review of instruments. Am J Psychiatry 2006; 163:1323-1334.

5 Vernillo A. Pragmatism as a complementary approach to legislation: closing regulatory gaps in human subject research. Am J Bioeth 2008; 8:15-17.

6 Koelch M, Singer H, Prestel A, et al. '... because I am something special' or 'I think I will be something like a guinea pig': information and assent of legal minors in clinical trials - assessment of 
understanding, appreciation and reasoning. Child Adolesc Psychiatry Ment Health 2009; 3:2.

7 Jacob R, Chowdhury AN. Assessment of mental capacity in patients recruited in clinical trials in psychiatry and its relationship to informed consent. Indian J Med Ethics 2009; 6:43-44.

8 Candalis PJ, Fletcher KE, Geppert CMA, et al. A direct comparison of research decision-making capacity: schizophrenia/schizo-affective, medically ill, and nonill subjects. Schizophr Res 2008; 99:350-358.

9 Okai D, Owen G, McGuire H, et al. Mental capacity in psychiatric patients. Br J Psychiatry 2007; 191:291-297.

10 Grisso T, Appelbaum PS. Assessing competence to consent to treatment: a guide for physicians and other health professions. New York: Oxford University Press; 1998.

11 Appelbaum PS, Grisso T. MacArthur competence assessment tool for clinical research. Sarasota, Florida: Professional Resource Press; 2001.

12 Owen GS, David AS, Richardson G, et al. Mental capacity, diagnosis and insight in psychiatric in-patients: a cross-sectional study. Psychol Med 2008; 22:1-10.

13 van Staden CW, Krüger C. Incapacity to give informed consent owing to mental disorder. J Med Ethics 2003; 29:41-43.

14 Stevenson GC, Ryan T, Anderson S. Principles, patient welfare and the Adults with Incapacity (Scotland) Act 2000. Int J Law Psychiatry 2009; 32:120-126.

15 Beauchamp TL, Childress JF. Principles of biomedical ethics. 5th ed. Oxford: Oxford University Press; 2001. pp. 160-162.

16 Adida M, Clark L, Pomietto P, et al. Lack of insight may predict impaired decision making in manic patients. Bipolar Disord 2008; 10:829-837.

17 Scott YHK. People admitted to psychiatric hospitals commonly lack the mental capacity to make treatment decisions. Evid Based Ment Health 2009; 12:31. 
18 Capdevielle D, Raffard S, Bayard S, et al. Competence to consent and insight in schizophrenia: is there an association? A pilot study. Schizophr Res 2009; 108:272-279.

19 Rutledge E, Kennedy M, O'Neill H, Kennedy HG. Functional mental capacity is not independent of the severity of psychosis. Int J Law Psychiatry 2008; 31:9-18.

20 Engh JA, Friis S, Birkenaes AB, et al. Delusions are associated with poor cognitive insight in schizophrenia. Schizophr Bull 2009 [Epub ahead of print]. The relationship was investigated in patients with schizophrenia between measurements on the Positive and Negative Syndrome Scale and measurements on the Beck Cognitive Insight Scale.

21 Jefferson AL, Lambe S, Moser DJ, et al. Decisional capacity for research participation in individuals with mild cognitive impairment. J Am Geriatr Soc 2008; 56:1236-1243.

22 Owen GS, Richardson G, David AS, et al. Mental capacity to make decisions on treatment in people admitted to psychiatric hospitals: cross sectional study. BMJ 2008; 337:a448.

23 Tan JOA, Doll HA, Fitzpatrick R, et al. Psychiatrists attitudes towards autonomy, best interests and compulsory treatment in anorexia nervosa: a questionnaire survey. Child Adolesc Psychiatry Ment Health 2008; 2:40.

24 Tumkaya S, Karadag F, Oguzhanoglu NK, et al. Schizophrenia with obsessive-compulsive disorder and obsessive-compulsive disorder with poor insight: a neuropsychological comparison. Psychiatry Res 2009; 165(1-2):38-46.

25 Raffin AL, Guimarães Fachel JM, Ferrão YA, et al. Predictors of response to group cognitivebehavioral therapy in the treatment of obsessive-compulsive disorder. Eur Psychiatry 2009; 24:297306.

26 Parellada M, Fraguas D, Bombín I, et al. Insight correlates in child- and adolescent-onset first episodes of psychosis: results from the CAFEPS study. Psychol Med 2009; 39:1433-1445.

27 Rabinovitch M, Béchard-Evans L, Schmitz N, et al. Early predictors of nonadherence to antipsychotic therapy in first-episode psychosis. Can J Psychiatry 2009; 54:28-35.

28 Patel MX, de Zoysa N, Bernadt M, David AS. A cross-sectional study of patients' perspectives 
on adherence to antipsychotic medication: depot versus oral. J Clin Psychiatry 2008; 69:1548-1556.

29 Llorca P. Partial compliance in schizophrenia and the impact on patient outcomes. Psychiatry Res 2008; 161:235-247.

30 Klingberg S, Schneider S, Wittorf A, et al. Collaboration in outpatient antipsychotic drug treatment: analysis of potentially influencing factors. Psychiatry Res 2008; 161:225-234.

31 Wittorf $A$, Jakobi $U$, Bechdolf $A$, et al. The influence of baseline symptoms and insight on the therapeutic alliance early in the treatment of schizophrenia. Eur Psychiatry 2009; 24:259-267.

32 Favrod J, Zimmermann G, Raffard S, et al. The Beck Cognitive Insight Scale in outpatients with psychotic disorders: further evidence from a French-speaking sample. Can J Psychiatry 2008; 53:783-787.

33 Palmer BW, Savla GN. The association of specific neuropsychological deficits with capacity to consent to research or treatment. J Int Neuropsychol Soc 2007; 13:1047-1059.

34 Simon V, De Hert M, Wampers M, et al. The relation between neurocognitive dysfunction and impaired insight in patients with schizophrenia. Eur Psychiatry 2009; 24:239-243.

35 Sanz M, Constable G, Loez-Ibor I, et al. A comparative study of insight scales and their relationship to psychopathological and clinical variables. Psychol Med 1998; 28:437-446.

36 Dunn LB, Palmer BW, Appelbaum PS, et al. Prevalence and correlates of adequate performance on a measure of abilities related to decisional capacities: differences among the standards of the MacCAT-CR in patients with schizophrenia. Schizophr Res 2007; 89:110-118.

37 Singer AR, Dobson KS. The effect of the cognitive style of acceptance on negative mood in a recovered depressed sample. Depress Anxiety 2009; 26:471-479.

38 Yen CF, Chen CC, Cheng CP, et al. Comparisons of insight in schizophrenia, bipolar I disorder, and depressive disorders with and without comorbid alcohol use disorder. Psychiatry Clin Neurosci 2008; 62:685-690. 